\title{
Mirror waves and mode transition observed in the magnetosheath by Double Star TC-1
}

\author{
J. Du ${ }^{1}$, C. Wang ${ }^{1}$, T. L. Zhang ${ }^{2}$, M. Volwerk ${ }^{2}$, and C. Carr ${ }^{3}$ \\ ${ }^{1}$ State Key Laboratory of Space Weather, Center for Space Science and Applied Research, Chinese Academy of Sciences, \\ Beijing, China \\ ${ }^{2}$ Space Research Institute, Austrian Academy of Sciences, 8042 Graz, Austria \\ ${ }^{3}$ The Blackett Laboratory, Imperial College, London, UK
}

Received: 15 December 2008 - Accepted: 13 January 2009 - Published: 22 January 2009

\begin{abstract}
The Double Star TC-1 magnetosheath pass on 26 February 2004 is used to investigate magnetic field fluctuations. Strong compressional signatures which last for more than an hour have been found near the magnetopause behind a quasi-perpendicular bow shock. These compressional structures are most likely mirror mode waves. There is a clear wave transition in the magnetosheath which probably results from the change of the interplanetary magnetic field (IMF) cone angle. The wave characteristics in the magnetosheath are strongly controlled by the type of the upstream bow shock.
\end{abstract}

Keywords. Magnetospheric physics (Magnetosheath; MHD waves and instabilities; Solar wind-magnetosphere interactions)

\section{Introduction}

TC-1 is the equatorial satellite of the geospace Double Star Project (DSP) which consists of two small satellites operating in the near-earth equatorial and polar regions. It was launched in December 2003 and finished its mission in October 2007. TC-1 had a large elliptical orbit with an apogee of about $13 R_{E}\left(1 R_{E}=6370 \mathrm{~km}\right)$. Since the apogee is rather far away from the Earth, TC- 1 crosses the bow shock and explores the whole magnetosheath. When the apogee of TC- 1 is on the day side, so is Cluster's, thus correlative observational studies can be made in the dayside magnetosheath. The Flux Gate Magnetometer (FGM, Carr et al.,

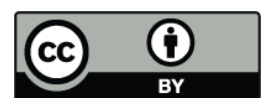

Correspondence to: J. Du

(jdu@spaceweather.ac.cn)
2005; Balogh et al., 2001) on board TC-1 and Cluster are used to measure the magnetic field.

The magnetosheath, which is often characterized by an ion temperature anisotropy $\left(T_{\perp}>T_{\|}\right)$and hot plasma conditions, is an ideal location for growth of low-frequency waves with angular frequency $\omega$ below or approximately at the proton gyrofrequency $\Omega_{p}$ (Sckopke et al., 1990). Mirror mode (MM) waves are commonly observed in the high-beta plasma of the magnetosheath (Song et al., 1992; Fazakerley and Southwood, 1994). MM is a compressible mode and exhibits linearly polarized magnetic field variations with wave vector nearly perpendicular to the background magnetic field (Pokhotelov et al., 2001a). Under the conditions of such a temperature anisotropy, another instability, namely electromagnetic ion cyclotron (IC) instability, can also grow to modest fluctuation level (Gary, 1992; Gary et al., 1993) in addition to mirror instability. The IC instability dominates in low $\beta$ plasma and generates transverse electromagnetic ion cyclotron waves propagating parallel to the magnetic field, however, the presence of He-ions suppresses the growthrate for proton IC waves (Gary et al., 1993).

MM waves are non-propagating modes in the plasma rest frame and compressive in nature. Lucek et al. (1999a,b) have identified mirror mode structures in the dawn-side magnetosheath from the Equator-S magnetic field data only. The relative change of the magnetic field strength, $\Delta B / B$, was used as a measure of the disturbance level in the field magnitude. Locally de-trended subsets of data points were used to calculate the angle between the local maximum variance direction and the average field direction. For MM waves this angle should be smaller than $30^{\circ}$ (Price et al., 1986) and the amplitude of fluctuations $\Delta B / B$ should be large. In this study we follow the same approach to identify mirror

Published by Copernicus Publications on behalf of the European Geosciences Union. 

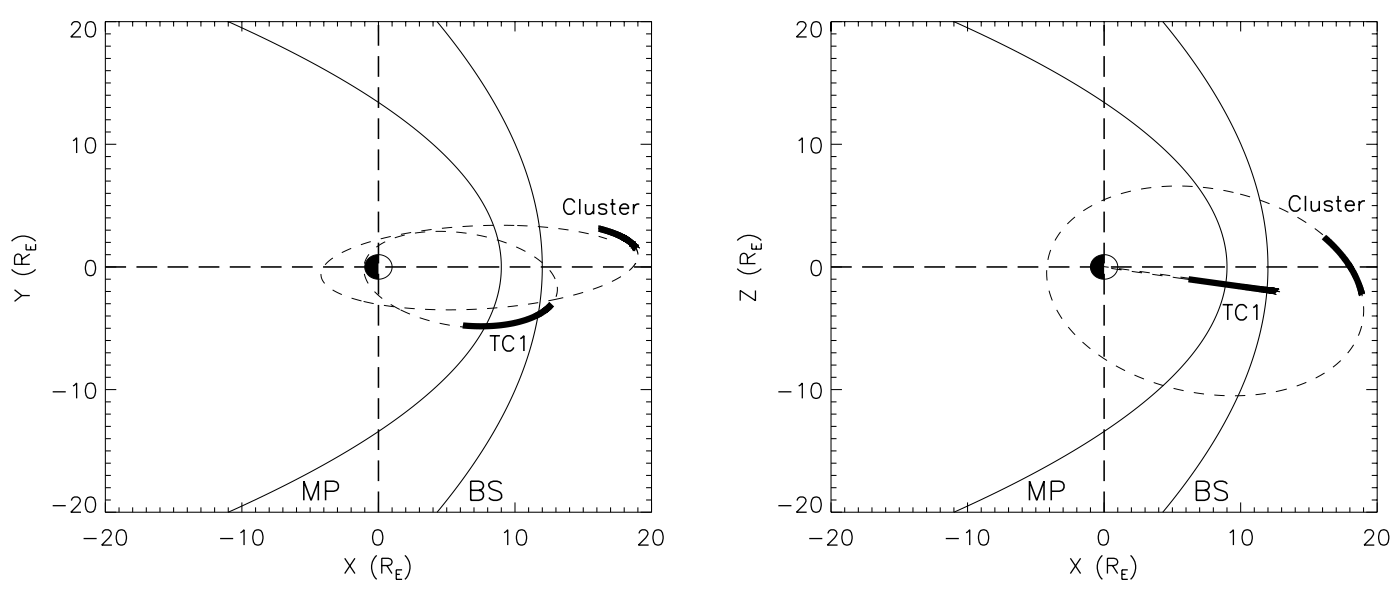

Fig. 1. Trajectories of TC-1 and Cluster projected to the GSE X-Y and X-Z planes for the interval 04:00-12:00 UT on 26 February 2004. Also shown are the nominal locations of the magnetopause (MP) and the bow shock (BS).

wave structures from the magnetic field data observed by TC-1 in the magnetosheath. Naturally, there are some other methods for mode identification (Song et al., 1994; Denton et al., 1995, 1998). But the available magnetic and plasma data are required by them, and unfortunately the plasma data from TC-1 for this case are not good enough. Therefore the method in Lucek et al. (1999a,b) which only used the magnetic field data became our best choice.

There are several possible sources for low frequency waves in the magnetosheath: solar wind fluctuations, foreshock waves, waves generated by the bow shock or the magnetopause, and waves that grow in the magnetosheath itself. Any change of these sources can result in wave-mode transition in the magnetosheath. A Large number of studies about the waves in the magnetosheath have been performed, which were reviewed in Schwartz et al. (1996), Song and Russell (1997) and Denton (2000). However, direct observations of wave-mode transitions in the magnetosheath are rarely reported in literature. In previous studies, ACE or Wind was used as a solar wind monitor, while other satellites were exploring the magnetosheath. Because of their large distance from Earth, it must take several dozens of minutes for the solar wind to flow from the monitors to Earth and thus it is hard to calculate the time delay accurately. In this study, while TC-1 was exploring the magnetosheath, Cluster was just in front of the bow shock at a distance of a few $R_{E}$. Therefore, it is easier to estimate the time delay from Cluster. We will study the influence of the upstream solar wind conditions on the magnetosheath properties. In this paper we will show that the change of upstream Interplanetary Magnetic Field (IMF) orientation results in an apparent transition of wave-modes in the magnetosheath.

\section{TC-1 observations and MM in the magnetosheath}

The data used in this study were collected by TC- 1 and Cluster between 04:00 and 12:00 UT on 26 February 2004. The orbits of TC-1 and Cluster for this period are shown in Fig. 1. The magnetopause and bow shock location is shown for average conditions of the solar wind. During this period TC1 made an outbound traversal of the magnetosheath from $(6.2,-4.8,-1.0) R_{E}$ to $(12.7,-3.0,-2.0) R_{E}$ GSE.

The magnetic data with a time resolution of $4 \mathrm{~s}$ obtained by TC- 1 and two parameters calculated from them are shown in Fig. 2. The panels from the top to bottom show the azimuth $(\phi)$ and elevation $(\theta)$ angles of the magnetic field in GSE coordinates, the magnetic field magnitude $|B|$, the fluctuation of the field $\triangle B / B$ and the angle between the maximum variance direction and average magnetic field $\alpha_{\mathrm{eB}}$, respectively.

As MM structures are characterized by compressional power with the fluctuations only in magnetic field magnitude and not in direction, it is preferable to present the magnetic field vector in polar coordinates. The azimuth angle $\phi$ is the angle between the projection of magnetic vector in $\mathrm{X}-\mathrm{Y}$ plane and the $X$-axis in GSE co-ordinates, while the elevation angle $\theta$ is the angle between the magnetic vector and the $X-Y$ plane. $\phi$ is measured counterclockwise from the positive half of the $\mathrm{x}$-axis to the projection of magnetic field and positive $\theta$ shows that the field has a northward component. To calculate the parameters $\Delta B / B$ and $\alpha_{\mathrm{eB}}, 2$ min sliding windows ( $\sim 30$ data points) with $1 \mathrm{~min}$ shift ( $\sim 15$ data points) were used. $\Delta B / B$ is the ratio of standard deviation to the average value of magnetic field. For each window, minimum variance analysis (Sonnerup and Scheible, 1998) was performed and the angle $\alpha_{\mathrm{eB}}$ between the maximum variance direction and the mean magnetic field was calculated.

The outbound magnetopause crossing at $\sim 05: 36$ UT can be easily identified by a sharp deviation of the magnetic field from the steady magnetospheric direction and a decrease in 


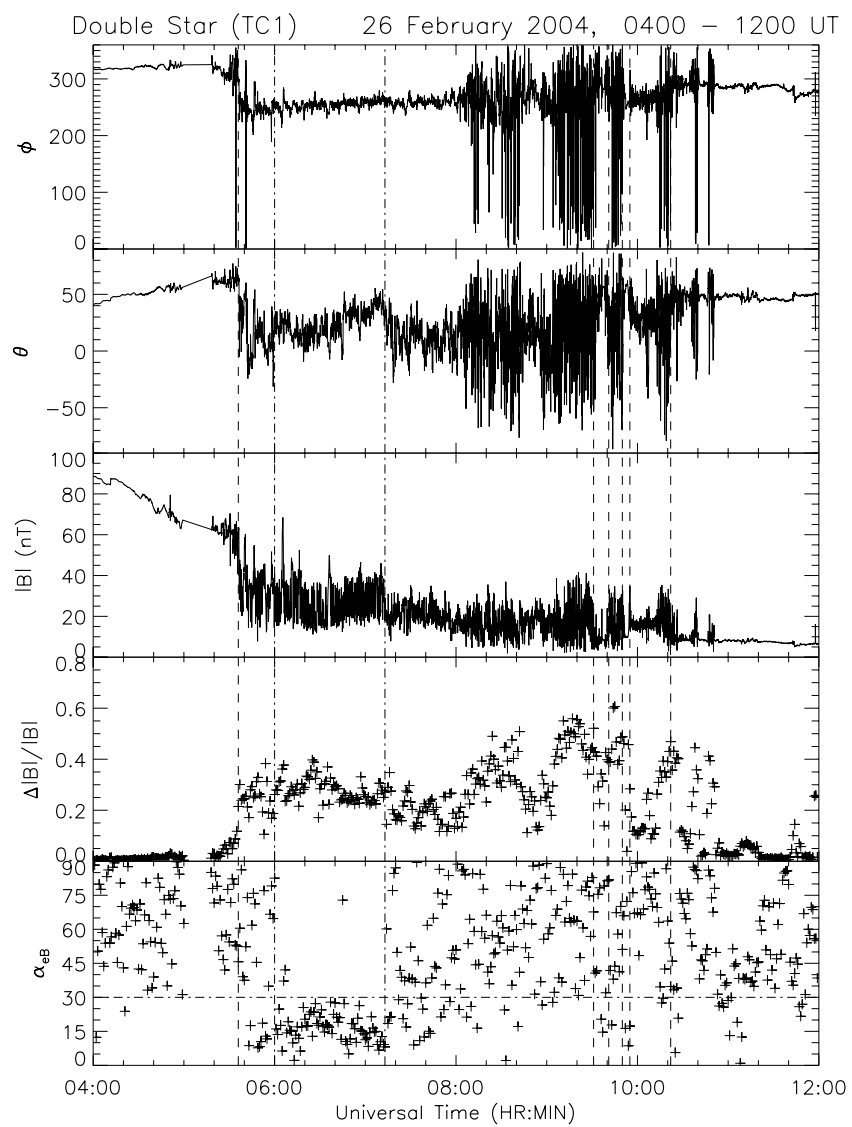

Fig. 2. Magnetic field data for the crossing of the magnetopause (first vertical dashed line at 05:38 UT), magnetosheath and bow shock (last 5 vertical dashed lines after 09:20 UT) observed by TC1 on 26 February 2004. From the top to bottom panels, shown are the azimuth $(\phi)$ and elevation $(\theta)$ angles of the magnetic field in GSE coordinates, magnetic field magnitude $|B|$, the fluctuation of the field $\Delta|\mathbf{B}| /|\mathbf{B}|$ and the angle between the maximum variance direction and average magnetic field $\alpha_{e B}$, respectively. The two vertical dash-dotted lines show the interval during which mirror mode waves are observed.

the field magnitude. It can be seen that there are several bow shock crossings at $\sim 09: 31,09: 41,09: 50,09: 55$, and 10:22 UT, respectively. The multiple bow shock crossings were probably due to the changing solar wind conditions. The positions of bow shock and magnetopause are marked with vertical dashed lines in Fig. 2.

Using the angle $\alpha_{\mathrm{eB}}$ in the bottom panel of Fig. 2, the interval from 06:00 to 07:13 UT can be identified by $\alpha_{e B} \leq 30^{\circ}$ and $\Delta B / B$ is relatively high $(\sim 0.3)$. This means that there are moderately intensive fluctuations of the magnetic field magnitude approximately parallel to the background field, and we identify that this region is dominated by MM waves.

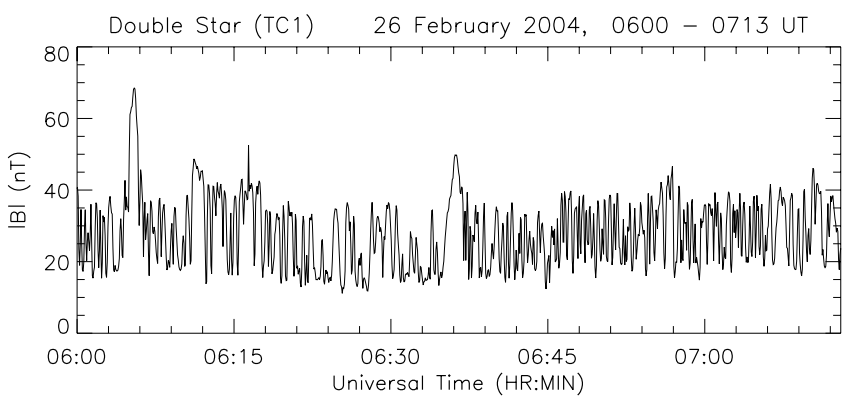

Fig. 3. The magnitude $|B|$ of magnetic file measured by TC-1 in the magnetosheath during the interval from 06:00 UT to 07:13 UT.

Figure 3 shows the magnetic field strength during the interval of MM-like activity from 06:00 to 07:13 UT. The strongly compressive fluctuations are dominant in this region. There are some larger amplitude and scale structures which interrupt the MM signatures (e.g. at $\sim 06: 06$ and $\sim 06: 36 \mathrm{UT}$ ). It has been suggested by Schwartz et al. (1996) that this type of large scale structures may evolve from MM structures after saturation. Such structures were also found by Lucek et al. (1999b) in the Equator-S observations.

\section{Wave-mode transition in the magnetosheath}

As shown in Fig. 2, the properties of the fluctuations changed sharply at $\sim 07: 13$ UT. Prior to this time the fluctuations were identified as MM. Afterwards, the field magnitude fluctuated less intensively and the compressional property was lost. There are many possible reasons for this wave mode transition, such as a change in local plasma conditions, increasing distance from magnetopause or changing upstream solar wind conditions. In this section, we will focus the influence of upstream IMF orientation on the wave mode in the magnetosheath.

As shown in Fig. 1, Cluster was in the solar wind region for this event. The magnitude, cone and clock angles of the IMF observed by Cluster are shown in Fig. 4. The cone angle is the angle between the IMF vector and the positive direction of $\mathrm{x}$-axis in GSE coordinates. The clock angle is the angle between the projection of the IMF in Y-Z plane and the positive $y$-axis (measured counterclockwise from the positive half of the $y$-axis). The two dash-dotted lines mark the time interval from 06:00 to 07:13 UT when TC-1 observed the MM signatures in the magnetosheath.

In panel 2 of Fig. 4 there is an apparent change of the cone angle from $\sim 90^{\circ}$ to $\sim 45^{\circ}$ at 07:05 UT, marked by a vertical dotted line. The two horizontal dashed lines indicate $45^{\circ}$ and $90^{\circ}$, respectively. The time when the IMF cone angle changed is close to the time of the wave-mode transition ( $\sim 07: 13 \mathrm{UT})$ in the magnetosheath. There is only a time delay of $\sim 8 \mathrm{~min}$ between them. If we are only considering the distance between two satellites and the solar wind speed of 


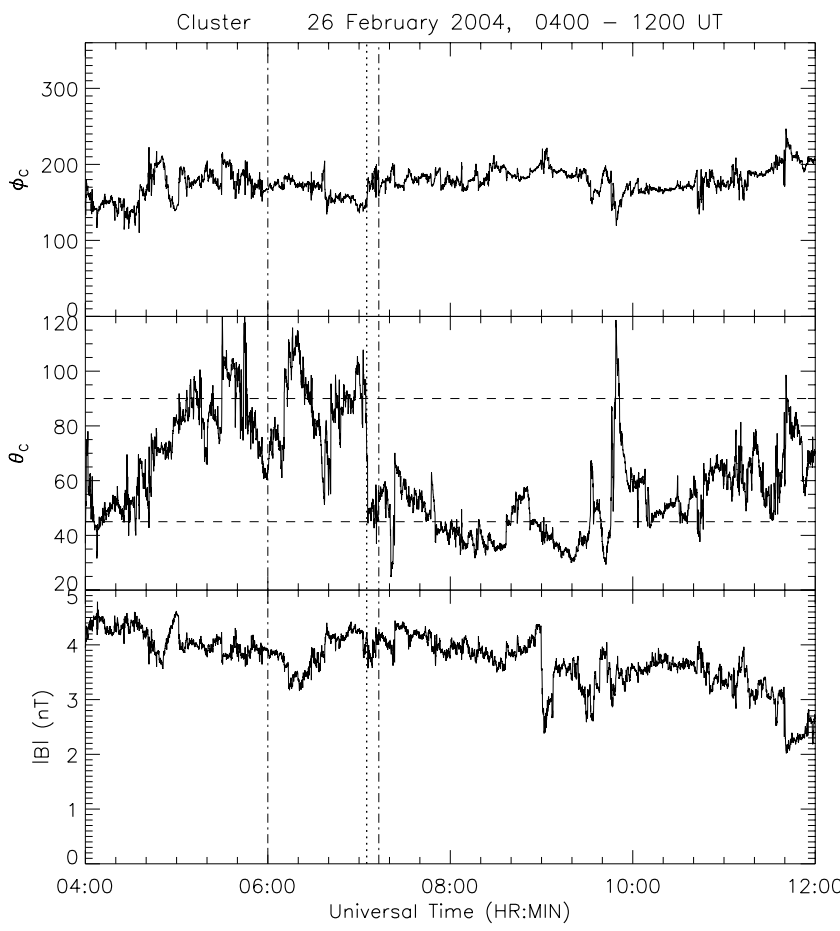

Fig. 4. The clock angle $\left(\phi_{\mathrm{c}}\right.$, top panel), cone angle $\left(\theta_{\mathrm{c}}\right.$, middle panel) and magnitude $|B|$ (bottom panel) of the IMF measured by Cluster.

$\sim 315 \mathrm{~km} / \mathrm{s}$, the time delay should be about $2.5 \mathrm{~min}$. However, the solar wind is decelerated at the bow shock and the actual time delay should be longer. The plasma speed measured by TC- 1 in the magnetosheath is $\sim 55 \mathrm{~km} / \mathrm{s}$. The distance between TC-1 and the bow shock can be determined easily by the location of the spacecraft at 07:13 UT and its position of bow shock crossing. Thus the revised time delay has been calculated and its value is $\sim 6 \mathrm{~min}$, which is still 2 min shorter than the observed value $(8 \mathrm{~min})$. This difference may result from: 1 . The distance and plasma speed were considered only along the $\mathrm{x}$-axis; 2 . The changing solar wind conditions might change the location of the bow shock; 3 . The plasma speed in the magnetosheath was strongly dependent on the relative position from the magnetopause. Near the time of mode transition there is no other clear change of the interplanetary conditions except IMF cone angle. Therefore, in spite of this difference of $2 \mathrm{~min}$ from the observed time delay, we think that the main reason for the wave mode transition in the magnetosheath is the change of IMF cone angle.

Because TC-1 was near the subsolar point in this case, the bow shock normal is approximatively parallel to the $X$-axis. Therefore, the IMF cone angle is nearly equal to the angle between the upstream magnetic field and the shock normal, $\theta_{B n}$, which is important for the generation of MM waves. MM waves are expected to occur during quasi-perpendicular shock conditions, which are often characterized by tempera- ture asymmetries created by e.g. shock-drift acceleration (see e.g. Kirk et al., 1994, Sect. 2.2). The IMF cone angle was $\sim 90^{\circ}$ before 07:05 UT and the TC-1 was observing the magnetosheath behind a quasi-perpendicular shock where MM waves were dominant. As the turn of the IMF, the property of the shock changed and the wave-mode transition in the downstream magnetosheath happened.

It is possible that the change of the shock property could impact the plasma properties in the magnetosheath, and then the different plasma conditions generate the different waves. However, the instrument HIA onboard TC-1 was saturated during this time interval and the plasma data in the magnetosheath were not available. It is impossible to estimate whether there is a large-scale spatial variation of plasma properties in the magnetosheath.

\section{Conclusions}

In this paper, a Double Star TC-1 magnetosheath pass on 26 February 2004 was used to investigate the magnetic field fluctuations, with emphasis on mirror-mode waves. The $\Delta B / B$ and $\alpha_{\mathrm{eB}}$ are used to identify the MM structures. The inner magnetosheath close to the magnetopause between 06:00 UT to 07:13 UT is dominated by compressional waves. While TC-1 was observing the fluctuations in the magnetosheath, Cluster was monitoring the interplanetary conditions just in front of bow shock at the same time. By comparing the properties of fluctuations in magnetosheath and the directions of IMF, we conclude that the IMF cone angle influences the downstream wave mode in this case. In the magnetosheath near subsolar point, the IMF cone angle is approximatively equal to the $\theta_{B n}$ of the upstream bow shock. As expected from theory the bow shock conditions (quasiperpendicular or quasi-parallel) determine the possible generation of MM waves in the magnetosheath. The region filled with the mirror waves was behind the quasi-perpendicular bow shock with $\theta_{B n} \approx 90^{\circ}$. When $\theta_{B n}$ decreases to $45^{\circ}$ and the upstream bow shock became quasi-parallel, the wave mode changed and the compressional property was virtually lost and no MM waves were observed anymore.

Acknowledgements. This work was supported by the Specialized Research Fund for State Key Laboratories, Chinese Academy of Sciences grant KJCX2-YW-T13 and the National Science Foundation of China (NSFC) grants 40674082, 40621003, and 40628003. The authors thank the CAA and the Cluster FGM team for the magnetic field data.

Topical Editor I. A. Daglis thanks S. P. Gary for his help in evaluating this paper.

\section{References}

Balogh, A., Carr, C. M., Acuña, M. H., et al.: The Cluster Magnetic Field Investigation: overview of in-flight performance and initial 
results, Ann. Geophys., 19, 1207-1217, 2001, http://www.ann-geophys.net/19/1207/2001/.

Carr, C., Brown, P., Zhang, T. L., et al.: The Double Star magnetic field investigation: instrument design, performance and highlights of the first year's observations, Ann. Geophys., 23, 2713 2732, 2005,

http://www.ann-geophys.net/23/2713/2005/.

Denton, R. E., Gary, S. P., Li, X. L., et al.: Low-frequency fluctuations in the magnetosheath near the magnetopause, J. Geophys. Res., 100, 5665-5679, doi:10.1029/94JA03024, 1995.

Denton, R. E., Lessard, M. R., LaBelle, J. W., et al.: Identification of low-frequency magnetosheath waves, J. Geophys. Res., 103, 23661-23676, doi:10.1029/98JA02196, 1998.

Denton, R. E.: ULF waves in the magnetosheath, Int. J. Geomag. and Aeron., 2, 44-55, 2000.

Fazakerley, A. N. and Southwood, D. J.: Mirror instability in the magnetosheath, Adv. Space Res., 14, 65-68, doi:10.1016/02731177(94)90049-3, 1994.

Gary, S. P.: The mirror and ion-cyclotron anisotropy instabilities, J. Geophys. Res., 97, 8519-8529, 1992.

Gary, S. P., Fuselier, S. A., and Anderson, B. J.: Ion anisotropy instabilities in the magnetosheath, J. Geophys. Res., 98, 14811488, 1993.

Kirk, J. G., Melrose, D. B., and Priest, E. R.: Plasma Astrophysics, Saas-Fee Advanced Course 24, Springer Verlag, Berlin, Germany, 1994.

Lucek, E. A., Dunlop, M. W., Balogh, A., et al.: Mirror mode structures observed in the dawn-side magnetosheath by Equator-S, Geophys. Res. Lett., 26, 2159-2162, doi:10.1029/1999GL900490, 1999.
Lucek, E. A., Dunlop, M. W., Balogh, A., et al.: Identification of magnetosheath mirror modes in Equator-S magnetic field data, Ann. Geophys., 17, 1560-1573, 1999, http://www.ann-geophys.net/17/1560/1999/.

Pokhotelov, O. A., Balikhin, M. A., Treumann, R. A., and Pavlenko, V. P.: Drift mirror instability revisited, 1 , cold electron temperature limit, J. Geophys. Res., 106, 8455-8463, 2001.

Price, C. P., Swift, D. W., and Lee, L. C.: Numerical simulation of nonoscillatory mirror waves at the earth's magnetosheath, J. Geophys. Res., 91, 101-112, 1986.

Schwartz, S. J., Burgess, D., and Moses, J. J.: Low-frequency waves in the Earth's magnetosheath: present status, Ann. Geophys., 14, 1134-1150, 1996, http://www.ann-geophys.net/14/1134/1996/.

Sckopke, N., Paschmann, G., Brinca, A. L., et al.: Ion thermalization in quasi-perpendicular shocks involving reflected ions, J. Geophys. Res., 95, 6337-6352, 1990.

Song, P., Russell, C. T., and Thomsen, M. F.: Slow mode transition in the frontside magnetosheath, J. Geophys. Res., 97, 82958305, 1992.

Song, P., Russell, C. T., and Gary, S. P.: Identification of lowfrequency fluctuations in the terrestrial magnetosheath, J. Geophys. Res., 99, 6011-6025, doi:10.1029/93JA03300, 1994.

Song, P. and Russell, C. T.: What do we really know about the magnetosheath, Adv. Space Res., 20, doi:10.1016/S02731177(97)00466-3, 747-765, 1997.

Sonnerup, B. U. Ö. and Scheible, M.: Minimum and maximum variance analysis, in: Analysis Methods for Multi-Spacecraft Data, edited by: Paschmann, G. and Daly, P., pp. 185-220, ESA, Noordwijk, 1998. 